\title{
Sensitive and Selective Label-free Alkaline Phosphatase Detection Based on DNA Hairpin Probe
}

\author{
Ziwei Zhao, Wenping Zhu, Zhen LI, Jianhui Jiang, ${ }^{\dagger}$ Guoli Shen, and Ruqin YU ${ }^{\dagger}$ \\ State Key Laboratory of Chemo/Bio-Sensing and Chemometrics, College of Chemistry and Chemical \\ Engineering, Hunan University, Changsha 410082, P. R. China
}

\begin{abstract}
Here, we present a label-free, simple and signal-on architecture for fluorescent alkaline phosphatase (ALP) biosensor utilizing an SYBR Green I (SG) assisted fluorescence amplification method. The strategy relies on the fact that ALP provides a significant barrier to lambda exonuclease ( $\lambda$ exo) activity by a dephosphorylating DNA hairpin probe (HP), and SG shows a considerable fluorescence intensity enhancement upon binding to double-stranded DNA (dsDNA) than single-stranded DNA (ssDNA). Our method is simple, sensitive and selective, which can also successfully detect the activity of ALP in complex biological fluids. The results have revealed that the method allows a specific and quantitative assay of the target ALP with a wide linear response range from 0.4 to $200 \mathrm{U} / \mathrm{mL}$ and a detection limit of $0.05 \mathrm{U} / \mathrm{mL}$.
\end{abstract}

(Received June 1, 2012; Accepted July 2, 2012; Published September 10, 2012)

\section{Introduction}

Alkaline phosphatase (ALP) is a hydrolase enzyme responsible for removing phosphate groups from many types of molecules, including nucleotides, proteins, and alkaloids. ALP plays crucial roles in many biological processes involving genetic transduction, cell metabolism and neuronal functions. These species are often used as biomarkers in the diagnosis of many diseases in clinical assays. ${ }^{1}$ Sensitive and selective detection of the ALP activity is of great value for monitoring of the differentiation of embryonic stem cells, the diagnosis of disease and the development of effective biomedical countermeasures. ${ }^{2-4}$

Actually, substantial efforts have been devoted to the development of rapid and sensitive analytical methods for detecting the activities of ALP. Different methods for monitoring the activities of ALP have been developed, such as radical isotope ${ }^{32} \mathrm{P}$-labeling, PAGE, and autoradiography. ${ }^{5-7}$ All of these methods, however, being laborious and time-consuming, and at the same time discontinuous and cost-ineffective, require radio labeling for sensitive detection. Up to now, new approaches using different techniques have also been reported, such as chemiluminescence, ${ }^{8} \quad$ colorimetry, ${ }^{9,10}$ fluorescence, ${ }^{11,12}$ electrochemistry ${ }^{13,14}$ and surface-enhanced Raman spectroscopy. ${ }^{15}$ However, some of these methods are time-consuming, or require complicated and expensive instruments; others can only obtain relatively weak signals and the operation steps are also labor-intensive. Therefore, the development of new analytical techniques for the rapid and convenient detection of ALP is of considerable interest.

Hairpin-shaped oligonucleotide probes have been widely applied to studying DNA-protein interactions, ${ }^{16}$ investigating genetic disease, ${ }^{17}$ and detecting nucleic acids in solution ${ }^{18}$ and living cells. ${ }^{19}$ Song et al. ${ }^{20}$ developed a high-throughput

† To whom correspondence should be addressed.

E-mail: jianhuijiang@hnu.edu.cn; rqyu@hnu.edu.cn screening platform for DNA 3 '-phosphatases and their inhibitors. The method was based on dephosphorylation and the polymerase reaction, which also involved the use of a molecular beacon and quantitative real-time PCR. Such a system was somehow complex and costly. Moreover, it offered a "signal off" mechanism, which might limit its applications. Therefore, there is a great need to develop more convenient, rapid and cost effective approaches for assaying ALP.

Here, we designed a DNA hairpin probe (HP) with a 5 -phosphoryl end for the sensitive and selective detection of ALP. The label-free strategy by means of lambda exonuclease ( $\lambda$ exo) cleavage was coupled with SYBR Green I (SG) assisted fluorescence amplification. The DNA HP with a $5^{\prime}$-phosphoryl end serves as a substrate for ALP. After the addition of target ALP, the $5^{\prime}$-phosphoryl end is hydrolyzed to yield a 5'-hydroxyl end that is immediately used to hamper the $\lambda$ exo cleavage, resulting in intact HP. With SG staining of the double-helix DNA of HP, a very high fluorescence enhancement is achieved. In the absence of ALP, $\lambda$ exo digests the phosphorylated HP into single-stranded DNA (ssDNA) and nucleotide fragments. SG shows weak fluorescence upon binding to ssDNA. Thus, the concentration of ALP is directly related to the fluorescence generated. This method was successfully applied to assay ALP in complex biological fluids.

\section{Experimental}

\section{Materials and chemicals}

SYBR Green I (10000×), fetal calf serum (FCS), plateletderived growth factor (PDGF)-BB, streptavidin (SA), human thrombin and GoldView ${ }^{\mathrm{TM}}$ Nucleic Acid Stain were obtained from Dingguo Biochemical Reagents (Beijing). Lysozyme was purchased from Sigma-Aldrich (St. Louis, MO). Calf intestinal alkaline phosphatase (CIP), lambda exonuclease ( $\lambda$ exo) and bovine serum albumin (BSA) were supplied by New England Biolabs Ltd. (Beijing). Sodium orthovanadate $\left(\mathrm{Na}_{3} \mathrm{VO}_{4}\right)$ was 
obtained from Sangon Biotechnology Co. Ltd. (Shanghai). The DNA oligonucleotides used in this study were synthesized by Sangon Biotechnology Co. Ltd. (Shanghai). The sequences of the oligonucleotides are shown as follows, where the loop sequences are in italics, and complementary sequences underlined:

\section{HP: 5'-phos-GAAGTATAAGGCGCGAAGTTTTCTTCGCGC CTTATACTTC-3'}

HP-1: 5'-GAAGTATAAGGCGCGAAGTTTTCTTCGCGCCT TATACTTC-3'

The buffer solutions employed in this work were as follows. The reaction buffer for CIP was $100 \mathrm{mM} \mathrm{NaCl}, 50 \mathrm{mM}$ Tris- $\mathrm{HCl}, 10 \mathrm{mM} \mathrm{MgCl}_{2}$ and $1 \mathrm{mM}$ dithiothreitol (pH 7.9). The reaction buffer for $\lambda$ exo was $67 \mathrm{mM}$ glycine- $\mathrm{KOH}, 2.5 \mathrm{mM}$ $\mathrm{MgCl}_{2}$ and $50 \mu \mathrm{g} / \mathrm{mL}$ BSA (pH 9.4). The detection buffer was $10 \mathrm{mM}$ Tris- $\mathrm{HCl}, 100 \mathrm{mM} \mathrm{NaCl}$, and $5 \mathrm{mM} \mathrm{MgCl}_{2}$ (pH 8.0). All other chemicals involved were of analytical-reagent grade. Deionized and sterilized water (resistance $>18 \mathrm{M} \Omega \mathrm{cm}$ ) was used throughout.

\section{Apparatus}

Fluorescence detection was carried out on a Hitachi F-7000 fluorescence spectrometer (Hitachi Ltd., Japan). The optical path length of a quartz fluorescence cell was $1.0 \mathrm{~cm}$. Excitation and emission slits were all set for a 5.0-nm band-pass, and $950 \mathrm{~V}$ was chosen as the excitation voltage. All optical measurements were performed under room temperature unless otherwise indicated.

Assay procedures

A CIP stock solution was firstly diluted to a series of concentrations from 1 to $2000 \mathrm{U} / \mathrm{mL}$ by using its reaction buffer. Then, the CIP solutions were incubated with $0.25 \mu \mathrm{M} \mathrm{HP}$ $(10 \mu \mathrm{L})$ at $37^{\circ} \mathrm{C}$ for $1 \mathrm{~h}$. $\lambda$ exo $(5 \mathrm{U}), 2.5 \mu \mathrm{L} 10 \times \lambda$ exo reaction buffer and sterilized water were added to a final reaction volume of $25 \mu \mathrm{L}$, and the reaction solutions were kept at $37^{\circ} \mathrm{C}$ for $30 \mathrm{~min}$. After that, the above-mentioned solutions were heated at $75^{\circ} \mathrm{C}$ for $10 \mathrm{~min}$ to inactivate CIP and $\lambda$ exo. Finally, $1 \mu \mathrm{L}$ of SG (100×) was added to the reaction solutions, and kept at room temperature for $10 \mathrm{~min}$. Detection buffer was added to give a final volume of $100 \mu \mathrm{L}$; the fluorescence was immediately measured and recorded. The excitation and emission wavelengths were set to 497 and $523 \mathrm{~nm}$, respectively.

To verify the feasibility of this strategy, an electrophoresis experiment was performed. Electrophoresis analysis was carried out on $4 \%$ agarose gels by GoldView staining, casting and running in $0.5 \times$ TBE buffer $(45 \mathrm{mM}$ Tris, $45 \mathrm{mM}$ boric acid, $1.25 \mathrm{mM}$ EDTA, pH 7.9) at room temperature. Electrophoresis was performed at a constant potential of $110 \mathrm{~V}$ for $90 \mathrm{~min}$ with the loading of $10 \mu \mathrm{L}$ of each sample into the lanes. The resulting gel was excited using a WD-9403F UV device and imaged with a Canon digital camera.

\section{Effect of inhibitor on the dephosphorylation reaction}

The proposed strategy can be further adapted to study the effects of inhibitor compounds on the phosphatase activity of CIP. $\mathrm{Na}_{3} \mathrm{VO}_{4}$ has been reported to be an inhibitor of CIP. ${ }^{14}$ The effect of $\mathrm{Na}_{3} \mathrm{VO}_{4}$ on the phosphatase activity of CIP was investigated according to following procedure: CIP $(200 \mathrm{U} / \mathrm{mL})$ was firstly mixed with varying concentrations of $\mathrm{Na}_{3} \mathrm{VO}_{4}$; then, the solutions were incubated with $0.25 \mu \mathrm{M} \mathrm{HP}(10 \mu \mathrm{L})$ at $37^{\circ} \mathrm{C}$ for $1 \mathrm{~h}$. The following experimental steps involving the addition of $\lambda$ exo, as well as the inactivation and addition of SG were the same as the aforementioned assay procedure for CIP.

We also investigated the interference of the assay by other

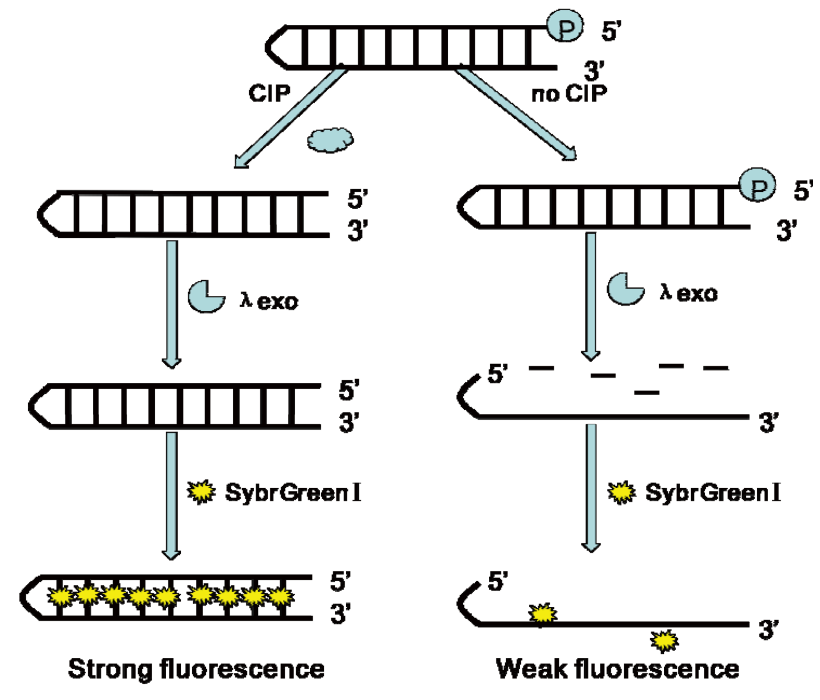

Scheme 1 Schematic illustrating the principle mechanism involved in the label-free fluorescent assay of CIP based on a DNA hairpin probe.

proteins, and evaluated the application in biological fluids. The experimental steps were performed according to the above procedure for inhibition with slight modifications (Supporting Information).

\section{Results and Discussion}

Design of a hairpin probe and alkaline phosphatase detection principle

The developed strategy for the label-free detection of ALP relies on the fact that the $\lambda$ exo activity is hampered by ALP through dephosphorylating 5 '-phosphoryl HP, and the staining of dephosphated HP with SG could result in great fluorescence enhancement. The analytical principle is illustrated in Scheme 1. The DNA oligonucleotide contains two single stranded self-complementary sequences that can form a hairpin probe having a 5'-phosphoryl end under appropriate hybridizing conditions. The $5^{\prime}$-phosphoryl HP formed in such a way offers a preferred substrate for $\lambda$ exo. $\lambda$ exo is a highly processive $5^{\prime} \rightarrow 3^{\prime}$ exodeoxyribonuclease that selectively digests the phosphorylated strand(s) of dsDNA. It was reported to degrade dsDNA with the $5^{\prime}$-phosphoryl end at a speed of 12 nucleotides/s, approximately, ${ }^{21,22}$ which is over 300 -times more quickly than that of dsDNA with the $5^{\prime}$-hydroxyl end. ${ }^{23}$ In the absence of CIP, the $5^{\prime}$-phosphoryl HP would be degraded by $\lambda$ exo, resulting in ssDNA with no secondary structure possible, leading to weak fluorescence intensity when stained with SG. Upon treatment with CIP, the HP with $5^{\prime}$-phosphoryl end is dephosphorylated into the $5^{\prime}$-hydroxyl end which is more slowly degraded by $\lambda$ exo, resulting in a great deal of intact HP. With SG staining of the double-helix DNA of HP, a strong fluorescence signal is generated. The CIP activity is quantified by monitoring the change in the fluorescence intensity.

To verify that the increase of the fluorescence signal was indeed caused by the binding of SG with double-helix DNA of dephosphated HP, and the later was obtained by dephosphorylating HP using ALP, an experiment for proof of principle was carried out. Another DNA hairpin probe, named as HP-1 (HP-1 has the same sequence as HP but lacking 


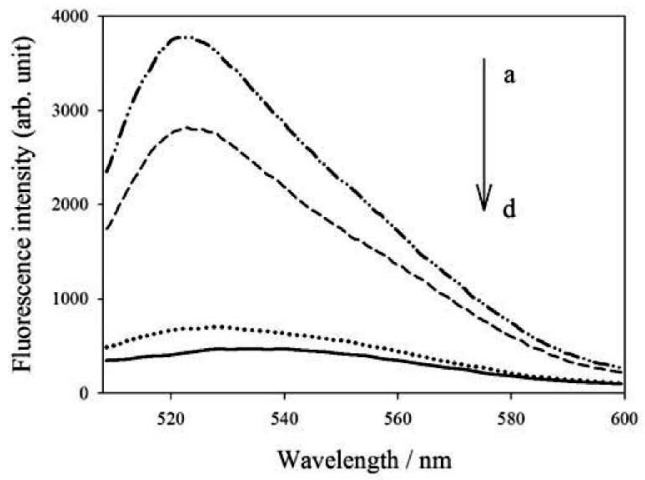

(A)

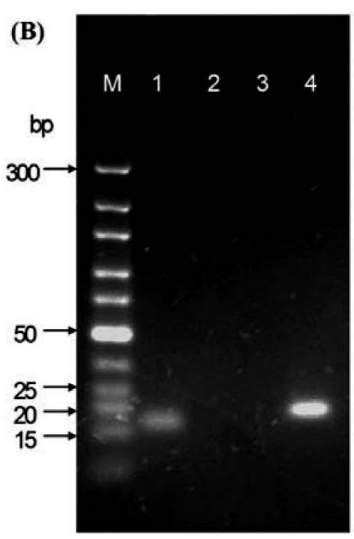

Fig. 1 (A) Proof of principle experiment to verify the feasibility of the proposed strategy. The fluorescence emission spectra under different conditions: (a) $100 \mathrm{nM} \mathrm{HP}-1$ digested by $5 \mathrm{U} \lambda$ exo and then stained with $1 \mu \mathrm{L} \mathrm{SG}(100 \times)$; (b) $100 \mathrm{nM}$ HP plus $2 \mathrm{U}$ CIP digested by $5 \mathrm{U} \lambda$ exo and then stained with $1 \mu \mathrm{L} \mathrm{SG}(100 \times)$; (c) $100 \mathrm{nM}$ HP digested by $5 \mathrm{U} \lambda$ exo and then stained with $1 \mu \mathrm{L} \mathrm{SG}$ (100×); (d) reaction buffer plus $1 \mu \mathrm{L} \mathrm{SG}(100 \times)$. (B) Electrophoresis analysis image for verifying the feasibility of the proposed strategy. Lane $1,0.6 \mu \mathrm{M}$ HP plus $4 \mathrm{U}$ CIP digested by $5 \mathrm{U} \lambda$ exo; Lane $2,0.6 \mu \mathrm{M} \mathrm{HP}$ digested by $5 \mathrm{U} \lambda$ exo; Lane 3, $0.6 \mu \mathrm{M}$ HP plus $1 \mu \mathrm{M}$ BSA digested by $5 \mathrm{U} \lambda$ exo; Lane $4,0.6 \mu \mathrm{M}$ HP-1 digested by $5 \mathrm{U} \lambda$ exo. M stands for DNA marker (O'GeneRuler ${ }^{\mathrm{TM}}$ Ultra-Low Range DNA Ladder, $10-300 \mathrm{bp})$.
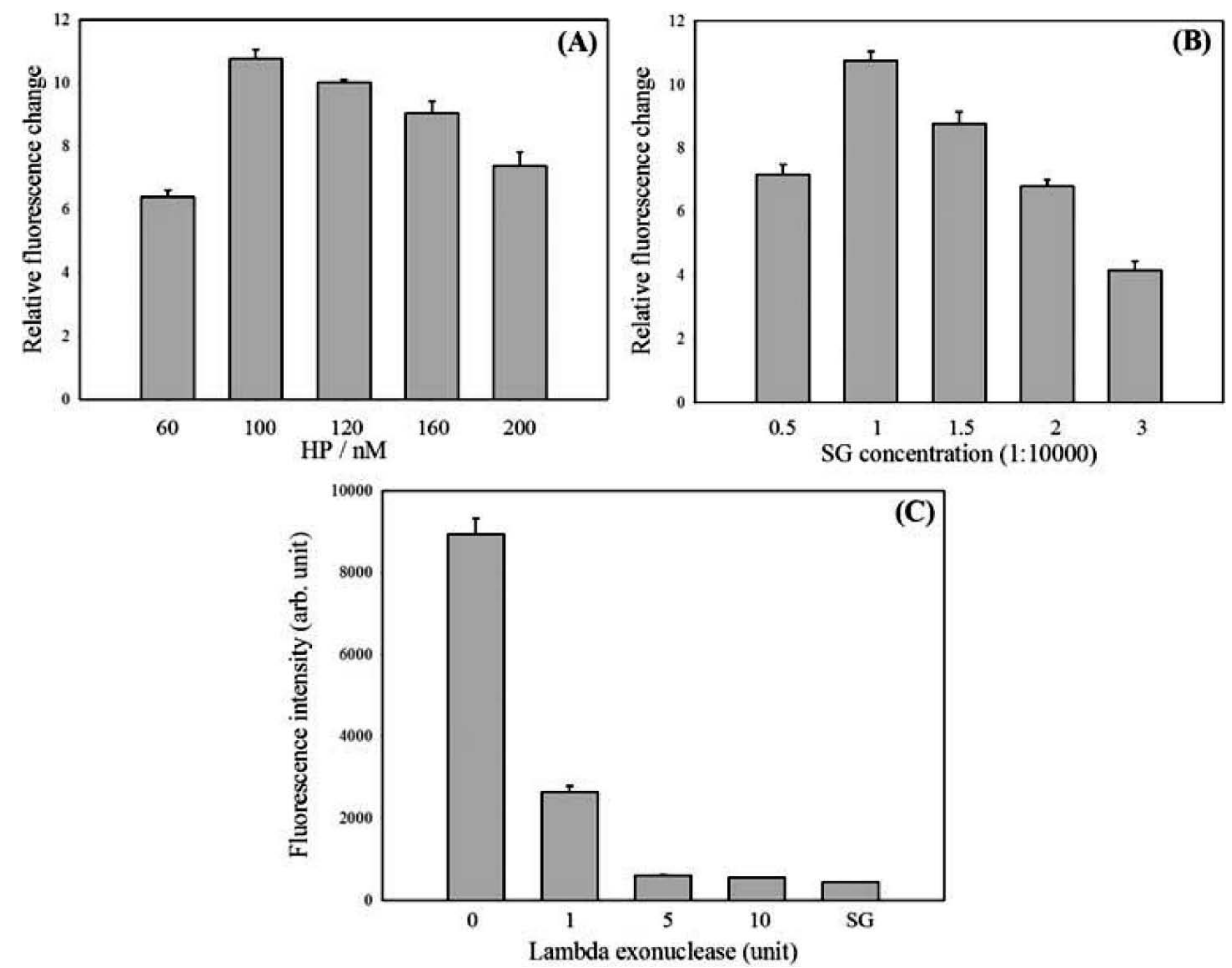

Fig. 2 Relative fluorescence changes in correlation to the experimental conditions. The relative fluorescence change is defined as $\left(F-F_{\mathrm{SG}}\right) /\left(F_{0}-F_{\mathrm{SG}}\right)$. (A) Dependency of the relative fluorescence changes on the concentration of HP. The concentrations of CIP, SG and $\lambda$ exo were $200 \mathrm{U} / \mathrm{mL}, \mathrm{SG}$ (1:10000) and $5 \mathrm{U}$, respectively. (B) Dependency of relative fluorescence changes on the concentration of SG. The concentrations of CIP, HP and $\lambda$ exo were $200 \mathrm{U} / \mathrm{mL}, 100 \mathrm{nM}$ and $5 \mathrm{U}$, respectively. (C) Dependency of relative fluorescence changes on the amount of $\lambda$ exo. The concentrations of CIP, HP and SG were $200 \mathrm{U} / \mathrm{mL}, 100 \mathrm{nM}$ and SG (1:10000), respectively. Bar "SG" stands for the fluorescence background of SG (1:10000).

5 -phosphoryl end), was used as a control. Figure 1A displays the fluorescence emission spectra under different conditions. As shown in Fig. 1A, in the absence of CIP, the HP was degraded into ssDNA and nucleotide fragments by $\lambda$ exo, and a weak fluorescence signal (curve c) was observed when stained with SG. However, in the presence of CIP, the HP with the 5 '-phosphoryl end was hydrolyzed by CIP to yield dephosphorylated HP that was resistant to $\lambda$ exo cleavage. 
After staining with SG, we observed a significant fluorescence signal (curve b). Since the HP-1 with 5 '-hydroxyl end was much more slowly degraded by $\lambda$ exo than that of HP, more intact double-helix DNA was produced after $\lambda$ exo cleavage, and as a result the highest fluorescence signal (curve a) was obtained when stained with SG. Additionally, the "curve d" stands for the fluorescence background of SG. These results confirmed the feasibility of the analytical principle.

To further verify the feasibility of the analytical principle, an electrophoresis experiment was performed. Figure 1B displays typical gel electrophoresis images observed both in the absence and presence of ALP. As shown in Fig. 1B, in the absence of CIP, HPs in lanes 2 and 3 were degraded by $\lambda$ exo into ssDNA residuals and nucleotides, and would migrate out of the gel. Therefore, no DNA band was observed in lanes 2 and 3 . A relatively bright band was observed in lane 1, where HP was resistant to $\lambda$ exo cleavage after the dephosphorylation of CIP. The HP-1 with 5 -hydroxyl end in lane 4 was much more slowly degraded by $\lambda$ exo than that of HP, resulting in a great deal of intact double-helix DNA that could be selectively stained by GoldView. Therefore, we could visualize the brightest band in lane 4. The gel electrophoresis analysis further confirmed the feasibility of the analytical principle.

\section{Optimization of reaction conditions}

Fluorescence responses of the ALP assay were highly dependent on the concentrations of HP, SG and $\lambda$ exo. In order to achieve the best assay performance, a series of systematic optimization experiments aiming at these concentrations were carried out to obtain the optimal experimental conditions. Figure 2A displays the relative fluorescence response at various HP concentrations (The relative fluorescence change is defined as $\left(F-F_{\mathrm{SG}}\right) /\left(F_{0}-F_{\mathrm{SG}}\right)$. Here, $F$ and $F_{0}$ are the fluorescence intensity at $523 \mathrm{~nm}$ in the presence and absence of CIP, respectively, $F_{\mathrm{SG}}$ stands for the fluorescence background of $\mathrm{SG}$ ). It was observed that the relative fluorescence response increased with an increase of the HP concentration at first, then gradually decreased after a concentration of $100 \mathrm{nM}$, which displayed a peak-shape dependency on the concentration of HP with a maximum achieved using $100 \mathrm{nM}$ HP. Therefore, $100 \mathrm{nM}$ HP was used throughout subsequent experiments.

The concentration of SG was directly related to the signal-to-background ratio. Figure $2 \mathrm{~B}$ displays the relative fluorescence response at various SG concentrations. As shown in Fig. 2B, the relative fluorescence response reached the maximum when the final SG concentration was diluted to 1:10000 (SG, $1 \times$ ). Thus, the optimal concentration of SG was chosen to be 1:10000 (SG, $1 \times$ ) and used throughout subsequent experiments.

Moreover, considering that the fluorescence generated was directly related to the cleavage of $\lambda$ exo, we also optimized the amount of $\lambda$ exo. As shown in Fig. $2 \mathrm{C}$, the fluorescence intensity decreased with the increase of $\lambda$ exo, and reached a plateau after using $5 \mathrm{U} \lambda$ exo. Therefore, the optimal amount of $\lambda$ exo was chosen to be $5 \mathrm{U}$, and used throughout subsequent experiments.

\section{Analytical performance of sensing system}

We chose the fluorescence emission from SG at $523 \mathrm{~nm}$ to evaluate the performances of the proposed strategy. The CIP activity was quantified based on the optimal experimental conditions. Figure 3 displays the fluorescence spectral response of label-free assay for CIP of varying concentrations. We observed dynamically increased fluorescence peaks in response to CIP of increasing concentrations within the range from 0.1 to
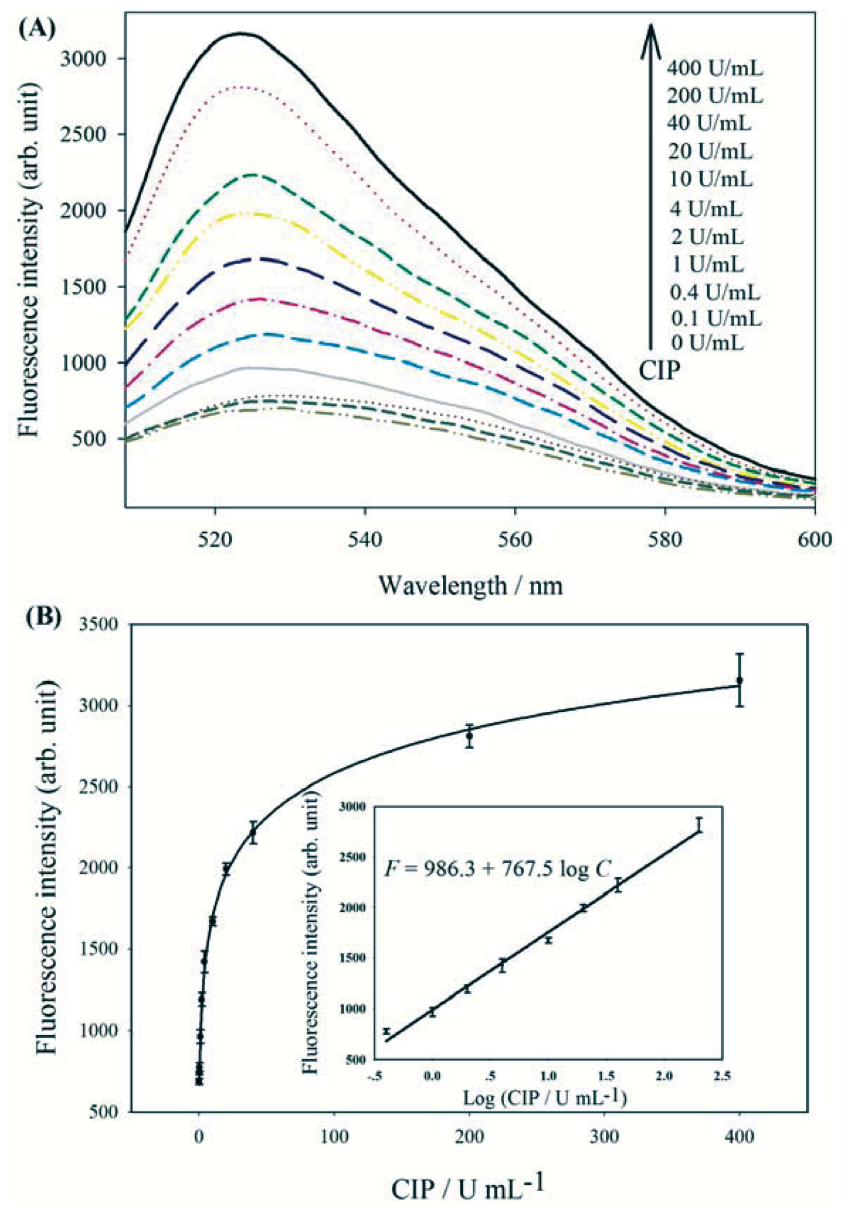

Fig. 3 (A) Fluorescence spectra response of the label-free assay for CIP of varying concentrations. Bottom to top: $0,0.1,0.4,1,2,4,10$, 20, 40, 200, $400 \mathrm{U} / \mathrm{mL}$. Experimental conditions were the optimal conditions. (B) Fluorescence spectra response at $523 \mathrm{~nm}$ versus CIP concentration (inset, dependence of fluorescence intensity on the logarithm of CIP concentration). The error bars represented for standard deviation (SD) across three repetitive experiments.

$400 \mathrm{U} / \mathrm{mL}$. There was a good linear correlation between the peak intensity and the logarithm of the CIP concentration in the range from 0.4 to $200 \mathrm{U} / \mathrm{mL}$. The linear relationship was $F=986.3+767.5 \log C$ ( $F$ is the fluorescence intensity and $\log C$ the logarithm of the CIP concentration) and the correlation coefficient was $R=0.997$. The detection limit was estimated to be $0.05 \mathrm{U} / \mathrm{mL}$ according to the $3 \sigma$ rule, which was lower than the previously reported electrochemical assay ${ }^{14}$ and comparable to the fluorescent assay based on universal molecular beacon and quantitative real-time PCR. ${ }^{20}$ The results demonstrated that the proposed strategy could be applied to sensitive ALP activity analysis over a wide concentration range.

\section{Interference of the ALP detection by inhibitor}

Many enzyme inhibitors are important compounds in biochemical studies and clinical diagnosis. Investigating the inhibition effect of enzyme inhibitors on the assay performance of the designed biosensor may provide a novel way to assay these biochemically or clinically important compounds. The proposed method can be further adapted to study the effect of an inhibitor on the phosphatase activity of CIP. The inhibitor used in this work was $\mathrm{Na}_{3} \mathrm{VO}_{4}$, which had no obvious effect on the activity of $\lambda$ exo under the experimental conditions (data not shown). 


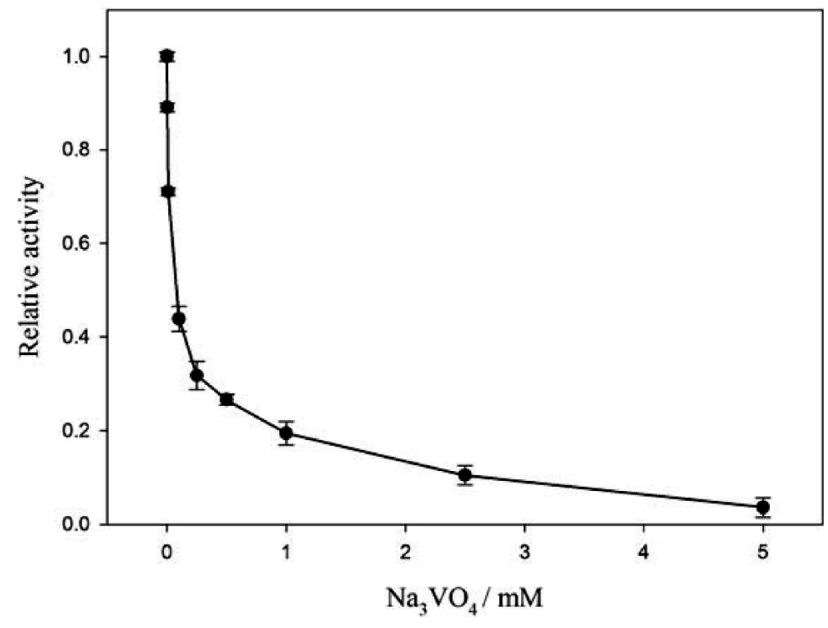

Fig. 4 Influence of $\mathrm{Na}_{3} \mathrm{VO}_{4}$ on the activity of CIP. The inhibition investigation was performed at varying concentrations of $\mathrm{Na}_{3} \mathrm{VO}_{4}: 0$, $0.0025,0.01,0.1,0.25,0.5,1,2.5$, and $5 \mathrm{mM}$.

Figure 4 displays the inhibition of CIP activity by $\mathrm{Na}_{3} \mathrm{VO}_{4}$. As shown in Fig. 4, the relative activity of CIP decreased sharply with the increasing concentrations of $\mathrm{Na}_{3} \mathrm{VO}_{4}$ up to $0.5 \mathrm{mM}$, and then gradually decreased until $5 \mathrm{mM}$ of $\mathrm{Na}_{3} \mathrm{VO}_{4}$ was used. $\mathrm{Na}_{3} \mathrm{VO}_{4}$ at concentration higher than $2.5 \mathrm{mM}$ can inhibit the phosphatase activity of CIP by more than $90 \%$.

\section{Selectivity of the ALP detection}

Achieving a high selective response to the analyte of interest over other interfering species is required for a successful biosensor. In this work, selectivity tests were conducted by using BSA, PDGF-BB, SA, thrombin and lysozyme as controls. Figure 5A depicts the selectivity of the label-free assay for CIP compared to other interfering proteins. As shown in Fig. 5A, all of these five interfering proteins with the same concentration of $0.5 \mu \mathrm{M}$ failed to cause any obvious fluorescence changes, which clearly contrasted with the significant fluorescence changes yielded when using $200 \mathrm{U} / \mathrm{mL}$ of CIP. This demonstrated that the proposed strategy had good selectivity for ALP detection.

\section{ALP assay in biological fluids}

In order to investigate whether the proposed strategy would become a promising method for the assay of ALP in real sample, we firstly verified that the proposed strategy could be used in complex biological fluids. We applied the sensing system in analyzing samples containing CIP in spiked $1 \%$ fetal calf serum. Figure 5B displays the fluorescence responses to CIP in its reaction buffer or spiked $1 \%$ fetal calf serum. As shown in Fig. 5B, if employed in fetal calf serum, the yielded fluorescence intensities agreed well with those from the same concentrations of CIP in its reaction buffer. This suggested that the proposed strategy could be applied in complex biological fluids with satisfactory results, and might become a promising method for assay of ALP in real sample.

\section{Conclusions}

In conclusion, we developed a label-free, simple and signal-on architecture for ALP detection based on a DNA hairpin probe coupled with intercalating dye. Using this method, we were able to detect CIP at a concentration as low as $0.05 \mathrm{U} / \mathrm{mL}$.
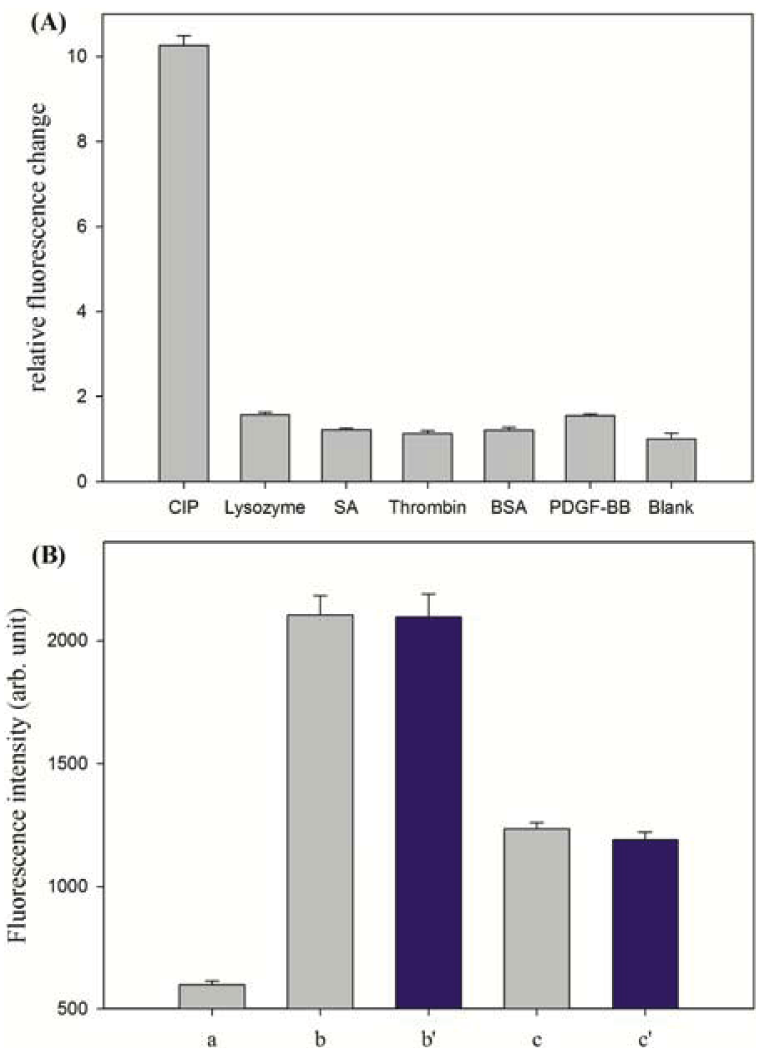

Fig. 5 (A) Selectivity of the proposed sensor for CIP compared to other interfering proteins. The concentration of CIP was $200 \mathrm{U} / \mathrm{mL}$. The concentrations of BSA, PDGF-BB, SA, thrombin and lysozyme were all $0.5 \mu \mathrm{M}$. The definition of the relative fluorescence change is the same as aforementioned in Fig. 2. (B) Fluorescence intensity obtained in the presence of CIP in the reaction buffer as well as spiked in $1 \%$ fetal calf serum. (a) Blank, (b) $40 \mathrm{U} / \mathrm{mL} \mathrm{CIP}$ in reaction buffer, (b') $40 \mathrm{U} / \mathrm{mL}$ CIP spiked in $1 \%$ fetal calf serum, (c) $4 \mathrm{U} / \mathrm{mL}$ CIP in reaction buffer, $\left(c^{\prime}\right) 4 \mathrm{U} / \mathrm{mL}$ CIP spiked in $1 \%$ fetal calf serum.

Higher sensitivity could be reached by extending the incubation time and stem length of the hairpin probe. We also investigated the interference of the CIP detection by inhibitor and other proteins as well as its capability of being applied to complex biological fluids. The proposed method is selective, sensitive and cost-effective, which may be promising to provide a general approach for alkaline phosphatase detection in analytical practice.

\section{Acknowledgements}

This work was supported by NSFC (21025521, 21035001, 21190041), National Key Basic Research Program (2011CB911000), CSIRT Program and NSF of Hunan Province (10JJ7002).

\section{Supporting Information}

This material is available free of charge on the Web at http:// www.jsac.or.jp/analsci/. 


\section{References}

1. C. Zhang, X. Su, Y. Liang, X. C. Zhu, C. Song, and M. P. Zhao, Biosens. Bioelectron., 2011, 28, 13.

2. I. Bronstein, C. S. Martin, J. J. Fortin, C. E. Olesen, and J. C. Voyta, Clin. Chem., 1996, 42, 1542.

3. D. Steiner, H. Khaner, M. Cohen, S. Even-Ram, Y. Gil, P. Itsykson, T. Turetsky, M. Idelson, E. Aizenman, R. Ram, Y. Berman-Zaken, and B. Reubinoff, Nat. Biotechnol., 2010 , 28, 361

4. P. L. J. Wolf, Clin. Lab. Anal., 1994, 8, 172.

5. L. K. Wang, C. D. Lima, and S. Shuman, EMBO J., 2001, 21,3873 .

6. K. Busheri, G. Daly, P. Robins, B. Canas, D. J. C. Pappin, J. Sgouros, G. G. Miller, H. Fakhrai, E. M. Davis, M. M. Le Beau, and M. Weinfeld, J. Biol. Chem., 1999, 274, 24187.

7. L. K. Wang and S. J. Shuman, J. Biol. Chem., 2001, 276, 26868.

8. V. E. Ximenes, A. Campa, W. J. Baader, and L. H. Catalani, Anal. Chim. Acta, 1999, 402, 99.

9. H. E. Grates, R. M. McGowen, S. V. Gupta, J. R. Falck, T. R. Brown, D. M. Callewaert, and D. M. Sasaki, J. Biosci., 2003, 28, 109.

10. H. Wei, C. G. Chen, B. Y. Han, and E. K. Wang, Anal.
Chem., 2008, 80, 7051.

11. S. Chen and M. P. Zhao, Anal. Chem., 2009, 81, 1383.

12. R. Freeman, T. Finder, R. Gill, and I. Willner, Nano Lett., 2010, 10, 2192.

13. X. M. Sun, N. Gao, and W. R. Jin, Anal. Chim. Acta, 2006, 571,30 .

14. P. Miao, L. M. Ning, X. X. Li, Y. Q. Shu, and G. X. Li, Biosens. Bioelectron., 2011, 27, 178.

15. C. M. Ruan, W. Wang, and B. H. Gu, Anal. Chem., 2006, 78, 3379.

16. J. J. Li, X. Fang, S. M. Schuster, and W. Tan, Angew. Chem., Int. Ed., 2000, 39, 1049.

17. S. Tyagi, D. P. Bratu, and F. R. Kramer, Nat. Biotechnol., 1998, 16, 49.

18. S. Tyagi and F. R. Kramer, Nat. Biotechnol., 1996, 14, 303.

19. D. L. Sokol, X. Zhang, P. Lu, and A. M. Gewirtz, Proc. Natl. Acad. Sci. U. S. A., 1998, 95, 11538.

20. C. Song, C. Zhang, and M. Zhao, Chem.-Asian J., 2010, $5,1146$.

21. K. Subramanian, W. Rutvisuttinunt, W. Scott, and R. S. Myers, Nucleic Acids Res., 2003, 31, 1585.

22. T. T. Perkins, R. V. Dala, P. G. Mitsis, and S. M. Block, Science, 2003, 301, 1914.

23. J. W. Little, J. Biol. Chem., 1967, 242, 679. 